\title{
An audit on the management of young people in an integrated community based sexual health clinic.
}

N Pal, S Ghodasara, M Hamill.

Berkshire Healthcare Foundation Trust. Garden Clinic, Slough.

Our community based integrated service caters to a diverse population within the Slough area with pockets of high deprivation and rising rates of CSE. It is of paramount importance that clinicians are able to identify and risk assess those vulnerable have a valuable tool for documentation. This audit was based on BASHH standards "Management of STIs and related conditions in children and young people (BASHH 2010)"

Methods: Retrospective data collection of the first 100 new patients who accessed the service from July - Aug 2014 aged 18 and under.

The standards of the audit are: Offer of full STI screen (CT, GC, HIV, STS) to sexually active young people $-100 \%$.

Uptake of STI screen-90\%.

Completions of CSE risk assessment proforma- $100 \%$.

Documentation of decision for referral ( $100 \%$ of under $13 \mathrm{~s}, 90 \%$ of those aged 16 and under)-100$90 \%$.

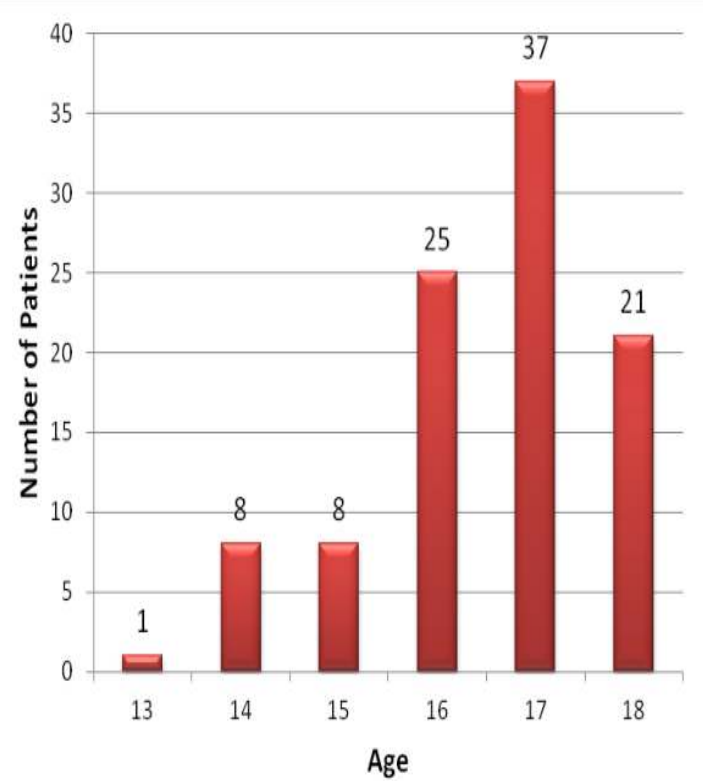

Age-wise distribution

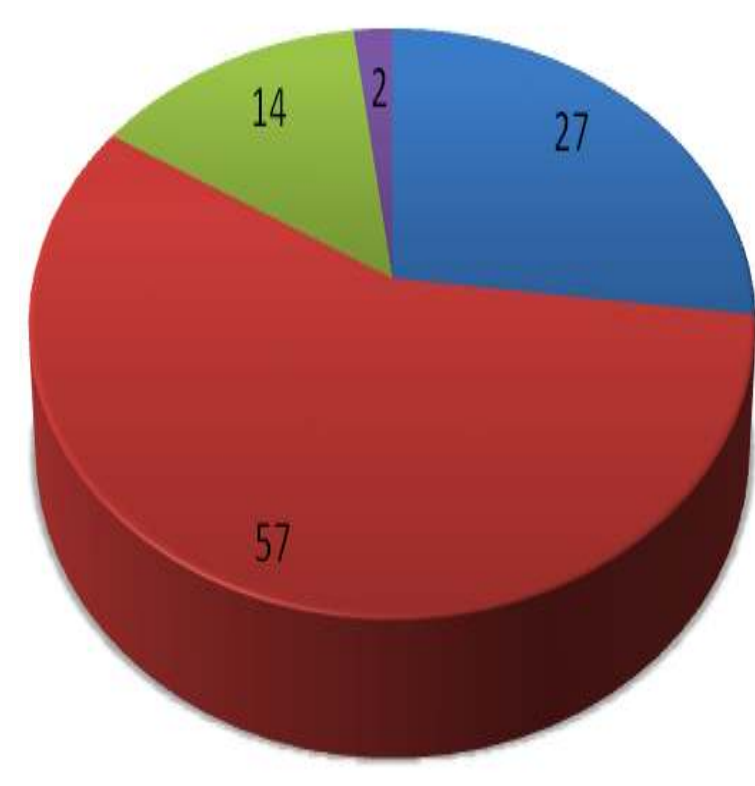

Reason for attendance

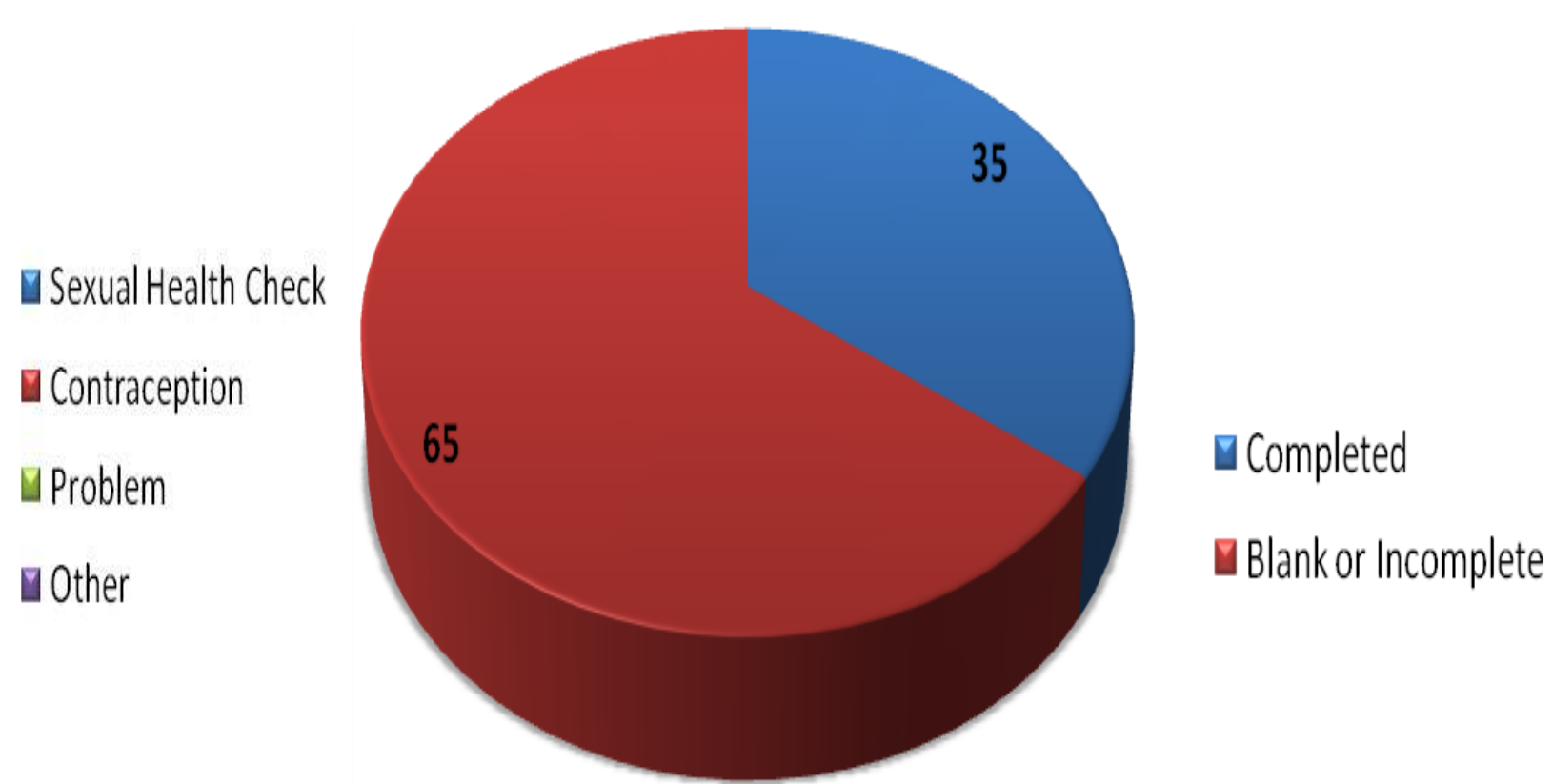

CSE proforma

Results: $75 \%$ of the attendees were girls and had primarily contraception needs. STI screening was offered to all however the uptake of a full screen was less than $50 \%$. Overall documentation was less than satisfactory and decision to refer was documented in only $15 \%$.

Conclusions: There are high rates of STI's among young people and risk taking behaviour was noticed in the attendances. Poor attendances among boys and MSM were identified. $50 \%$ refused to have screening for BBV and the need of alternative testing methods like the saliva testing was highlighted. A CSE proforma was introduced and all referrals are discussed with the safeguarding lead and audited on a regular basis. 